\title{
Direct purification of CRISPR/Cas ribonucleoprotein from E. coli in a single step
}

Siyu $\operatorname{Lin} \dagger^{1}$, Jie Qiao $\dagger^{1,2}$, Lixin $\mathrm{Ma}^{1,2}, \mathrm{Yi} \mathrm{Liu}^{1,2} *$

1. Hubei Collaborative Innovation Center for Green Transformation of Bio-resources, Hubei University, Wuhan, 430062 Hubei, China.

2. Hubei Key Laboratory of Industrial Biotechnology, School of Life Sciences, Hubei University, Wuhan, 430062 Hubei, China.

$\dagger$ These authors contributed equally to this work. Correspondence should be addressed to Y. Liu (yiliu0825@ hubu.edu.cn).

\begin{abstract}
CRISPR/Cas ribonucleoprotein (RNP) complexes have been recently used as promising biological tools with plenty of applications, however, there are by far no efficient methods to prepare them at large scale and low cost. Here, we present a simple method to directly produce and purify Cas RNP, including the widely used Cas9 and Cas12a nuclease, from E.coli in a single step using an ultra-high-affinity CL7/Im7 purification system. The prepared Cas RNP shows high stability, solid nuclease activity in vitro, and profound genome editing efficiency in vivo. Our method is convenient, cost-effective, and applicable to prepare other CRISPR associated nucleases.
\end{abstract}

\section{Keywords}

CRISPR, ribonucleoprotein, Cas9, Cas12a, CL7/Im7

\section{Introduction}

Clustered regularly interspaced short palindromic repeats (CRISPR)/CRISPR associated-protein (Cas) genome-editing systems, including the widely used 
Streptococcus pyogenes Cas9 (SpCas9) (Jinek et al. 2012; Koonin et al. 2017) and Francisella novicida Cas12a (previously named FnCpf1) nuclease (Fonfara et al. 2016; Zetsche et al. 2015), have been extensively applied to biomedical area with great promises to revolutionize the treatment of genetic diseases (Cho et al. 2013; Scott and Zhang 2017). Gene therapy with adeno-associated viruses (AAVs) is currently the most advanced approach for delivering Cas enzymes in vivo (Wu et al. ; Yin et al. 2016), though this methodology has limitations such as pre-existing immunity towards AAV in human and small packing size (Schumann et al. 2015). Recently, several researches demonstrated that direct delivery of CRISPR/Cas ribonucleoprotein (RNP) for genome editing in cells and animals has obvious advantages (Gao et al. 2018; Staahl et al. 2017; Yin et al. 2014), such as reduced off-target effects, low toxicity, high editing efficiency, etc. Thus, increasing biopharmaceutical companies are paying greater emphasis on developing Cas RNP-based gene therapy medicines.

The current strategy to construct Cas RNP needs to produce recombinant CRISPR-associated nuclease and the guide RNA (gRNA) individually, followed by assembling them in vitro (Kim et al. 2014). However, the preparation of pure RNP is always time-consuming and expensive (Anders and Jinek 2014). Therefore, the development of an efficient and cost-effective method to produce and purify Cas RNP with high yield and high purity remains a central problem in the field of RNP-based therapeutic gene editing.

In our recent work, we successfully achieved co-expression of Cas9 and guide RNA in E. coli to directly prepare Cas9 RNP in vivo (Li et al. 2018). We harnessed two chromatographic steps to purify the Cas9 RNP, including the first affinity purification by Ni-NTA column and the second purification by gel filtration. By the method, it takes us more than three days to prepare pure Cas9 RNP with a relatively low production $(\sim 10 \mathrm{mg} / \mathrm{L})$, which is still not yet satisfied with large-scale demand in industry. In this report, we utilize an ultra-high-affinity CL7/Im7 purification system (Vassylyeva et al. 2017) developed recently to carry out one-step purification of Cas RNP, including Cas9 and Cas12a, with great purity and improved yield ( $40 \mathrm{mg} / \mathrm{L}$ 
for Cas9; $50 \mathrm{mg} / \mathrm{L}$ for Cas12a). Meanwhile, the whole production process was largely reduced to half a day. The $\operatorname{Im} 7$ affinity column used in the work was easily made by ourselves through coupling the recombinant $\operatorname{Im} 7$ to agarose beads, and repeatedly regenerated without losing its affinity to the CL7 affinity tag. Together, we establish a valid platform to readily produce CRISPR-associated RNP at large-scale and low cost.

\section{Materials and methods}

Co-expression vector design and construction

We ordered CL7 gene form Sangon Biotech (Shanghai) Company and assembled it in the $\mathrm{N}$ - terminal of Cas9 or Cas 12 a with a TEV proteinase cleavage site between them. In addition, we preserved the C-terminal $6 \mathrm{x}$ His tag for purifying CL7-tagged fusion enzymes if required. Then, the CL7-Cas9 or CL7-Cas12a fragment was cloned into an engineered cold-shock vector pCold I (Takara) (Qing et al. 2004) by replacing the original cspA promoter with a tac promoter, and by replacing the f1 ori with a T7 promoter (Fig 1.). In addition, the vector contains a gRNA transcription element which can be easily replaced by SalI digestion. We generated two co-expression plasmids termed pCold CL7-Cas9 and pCold CL7-Cas12a, respectively.

Preparation of $\operatorname{Im} 7$ ligated agarose beads

The $\operatorname{Im} 7$ gene was synthesized by Sangon Biotech (Shanghai) Company and cloned into an pET23a(+) expression vector (Vassylyeva et al. 2017). The $\operatorname{Im} 7$ protein was overexpressed in E. coli BL21(DE3) cells and purified according to the protocol. Then, the prepared $\operatorname{Im} 7$ was covalently cross-linked to $1 \mathrm{mM}$ iodoacetyl agarose beads purchased from Sigma-Aldrich. After cleavage and elution of a target protein, the CL7-tag, which remained bound to the $\operatorname{Im} 7$ agarose beads, was removed under denaturing conditions (6 M Guanidine hydrochloride, Sinopharm Group Co.). The column was then easily regenerated via in-column $\operatorname{Im} 7$ refolding. 
Purification of Cas RNP by $\operatorname{Im} 7$ column

The co-expression plasmids constructed above were transformed into the E. coli Rosetta (DE) 3 and the cells were cultured at $37^{\circ} \mathrm{C}$ under $220 \mathrm{rpm}$ rotate speed. 0.5 mM IPTG was added when the $\mathrm{OD}_{600}$ reached 0.8 , and the temperature was decreased to $16^{\circ} \mathrm{C}$ for expression of Cas RNP. The cells were harvested and lysated in lysis buffer (20 mM Tris-HCl, pH 7.4, $100 \mathrm{mM} \mathrm{NaCl}$ ). The CL7 tagged Cas9 or Cas12a was loaded onto the $\operatorname{Im} 7$ column. After washing two cycles of washing buffer I (20 $\mathrm{mM}$ Tris- $\mathrm{HCl}, \mathrm{pH} 7.4,300 \mathrm{mM} \mathrm{NaCl}$ ), TEV protease was added to the column for in-column cleavage of CL7 tag for three hours. Next, the highly purified Cas9 or Cas12a protein was eluted with washing buffer II (20 mM Tris-HCl, pH 7.4, $500 \mathrm{mM}$ $\mathrm{NaCl})$. Finally, the Cas RNP was concentrated and stored at $-80^{\circ} \mathrm{C}$ in the storage buffer (20 mM Tris-HCl, pH 7.4, 500 mM NaCl, 20\% glycerin).

In vitro nuclease activity assay

For in vitro endonuclease activity test, the purified Cas RNP was directly applied to digest dsDNA. The digestion was typically carried out in a $10 \mu \mathrm{l}$ reaction mixture, composed of $1 \mu \mathrm{l} 10$ x buffer 3.1 (NEB), 200 ng Cas9 or Cas12a RNP, 300 ng plasmid, at $37^{\circ} \mathrm{C}$ for 30 minutes followed by termination of the reaction at $80^{\circ} \mathrm{C}$ for 10 minutes.

In vivo genome editing efficiency assay

For illustrating in vivo and homology dependent repair (HDR) efficiency, we adopted a BFP-expressing HEK293 reporter cell line according to the reported protocol (DeWitt et al. 2017; Lee et al. 2017). To edit BEP-HEK to GFP, the donor dsDNA and Cas9 RNP were co-delivered by lipofectamine CRISPRMAX (Yu etal. 2016) (ThemoalFisher). The HDR efficiency were calculated from the GFP cell counts/total cell counts (DeWitt et al. 2017; Lee et al. 2017). 


\section{Results}

Direct expression of CL7 tagged Cas RNP in E. Coli

To prepare a large amount of Cas RNP, we introduce a N-terminal CL7-tag (Vassylyeva et al. 2017) into the co-expression vector engineered from a commercial pCold I vector (Takara) (Qing et al. 2004) (Fig. 1). CL7 is a catalytically inactive variant of Colicin E7 (CE7) DNase with a pretty low $\mathrm{K}_{\mathrm{d}}$ $\left(\sim 10^{-14}-10^{-17} \mathrm{M}\right)$ towards its binding partner Immunity Protein $7(\operatorname{Im} 7)$ (Ko et al. 1999). The CL7/Im7 system has been reported recently to facilitate purification of diverse proteins (Vassylyeva et al. 2017). According to the design, the gRNA molecules were abundantly transcribed in vivo by $E$. coli's own RNA polymerases, while the CL7-Cas9 fusion proteins were largely produced when induced by IPTG. We have proved that the newly synthesized Cas 9 and transcribed gRNA molecules would be spontaneously self-assembled in $E$. coli cells to form complete Cas9/gRNA complexes (Li et al. 2018). Likewise, in this case, the CL7 tagged Cas9 would interact with the transcribed gRNA in vivo to form CL7-Cas9 RNP. Introduction of CL7 tag would not only significantly simplify the purification procedures, but also can hugely improve the solubility and yield of target proteins (Vassylyeva et al. 2017). As a result, it brings about five-fold increase in production of Cas9 RNP, compared to the method in absence of CL7 tag. In addition, we applied the method to produce Cas12a nuclease, and observed approximately ten-fold yield compared to the current method that used maltose binding protein (MBP) as fusion tag. More importantly, we found that the Cas9 (or Cas12a) with and without CL7 tag have the same endonuclease activity (Fig. 3a), possibly due to CL7 has a small molecular weight $(\sim 16 \mathrm{kD})$.

Purification of pure Cas RNP in a single step

We previously harnessed multiple chromatographic steps to obtain pure Cas9 RNP, therefore, a large number of Cas9 enzymes were lost during the 
purification procedures. In this work, following an optimized protocol (see SI for details), we successfully achieved purification of Cas RNP in a single step within half a day by the ultra-high-affinity CL7/Im7 system. Comparing to the Cas9 RNP purified by single Ni-NTA column, the purity of Cas9 RNP prepared by $\operatorname{Im} 7$ column was significantly improved (Fig. 2). More importantly, the $\operatorname{Im} 7$ affinity column was easily made (SI) by ourselves through coupling the recombinant $\operatorname{Im} 7$ proteins to agarose beads, and repeatedly regenerated without losing its affinity to the CL7 affinity tag, largely reducing the cost of materials.

The RNP prepared by the in vitro method currently used (Cho et al. 2013) is very unstable so that a sufficient amount of RNase inhibitors are required to prevent degradation of gRNA. By our co-expression method (Li et al. 2018), there is no needs to add RNase inhibitors in the whole purification and storage procedures. Herein, we found that the Cas9 or Cas12a RNP made by our in vivo method is also pretty stable that can maintain full nuclease activity when storing at $-20^{\circ} \mathrm{C}$ for up to half a year.

Characterization of the purified Cas RNP

To determine their in vitro nuclease activity, we observed that $300 \mathrm{ng}$ plasmids (Fig. 3a) were cleaved in less than 30 minutes at $37^{\circ} \mathrm{C}$ when $200 \mathrm{ng}$ of RNPs were added (SI). Accordingly, Cas9, CL7-Cas9 and Cas12a RNP can fully cleave the target plasmids. The endonuclease activity of our purified RNP is comparable to that of commercial Cas enzyme, indicating that the method can be industrially applicable to produce CRISPR-associated RNP.

To illustrate the in vivo homology dependent repair (HDR) efficiency of purified Cas9 RNP, we constructed an engineered BFP-HEK293 cell line as the reporter system (DeWitt et al. 2017; Lee et al. 2017). In principle, the BFP-HEK293 cells would be converted to GFP-expressing cells after co-delivering Cas9 RNP and donor dsDNA by lipofectamine CRISPRMAX (Fig. 3b). The determined HDR efficiency of our purified Cas9 RNP is about 
$20 \%$, which is comparable to the RNP made by in vitro as previously reported (Fig. 3c) (DeWitt et al. 2017; Lee et al. 2017).

\section{Discussion}

Recently, direct delivery of pre-formed Cas9 RNP has been proven as a promising method for genome editing with great advantages (Cho et al. 2013; DeWitt et al. 2017; Staahl et al. 2017; Yu and Liang 2016). However, it is often time-consuming and expensive to prepare Cas9 RNP in sufficient amounts by the current method through roughly mixing Cas 9 and gRNA in vitro. $\mathrm{n}$ conclusion, we utilize an easily removeable CL7 fusion tag to overexpress Cas9 or Cas12a RNP in vivo in E. coli, enabling significantly improved production of RNP. By harnessing the ultra-high-affinity CL7/Im7 purification system, we achieved one-step purification of pure Cas RNP with profound nuclease activity in vitro and in vivo, indicating that the method has great potentials to prepare CRISPR-associated nucleases at large-scale, low cost and short time.

\section{Acknowledgments}

We thank T.A. Liu for kindly gifting the genes involving astaxanthin biosynthetic pathway. This research was supported by Key Technical Innovation Foundation of Hubei Province (NO.2017ACA174 to L.X. Ma) and National Foundation of Hubei Province (NO. 201700963 to Y. Liu).

\section{Conflict of interest}

All authors in this manuscript have declared no conflict of interest。

\section{References}

Anders C, Jinek M (2014) In vitro enzymology of Cas9. Methods Enzymol 546:1-20

Cho SW, Kim S, Kim JM, Kim JS (2013) Targeted genome engineering in human cells with the Cas9 RNA-guided endonuclease. Nat Biotechnol 31(3):230-2 
DeWitt MA, Corn JE, Carroll D (2017) Genome editing via delivery of Cas9 ribonucleoprotein. Methods 121-122:9-15

Fonfara I, Richter H, Bratovic M, Le Rhun A, Charpentier E (2016) The CRISPR-associated DNA-cleaving enzyme Cpf1 also processes precursor CRISPR RNA. Nature 532(7600):517-21

Gao X, Tao Y, Lamas V, Huang M, Yeh WH, Pan B, Hu YJ, Hu JH, Thompson DB, Shu Y, Li Y, Wang H, Yang S, Xu Q, Polley DB, Liberman MC, Kong WJ, Holt JR, Chen ZY, Liu DR (2018) Treatment of autosomal dominant hearing loss by in vivo delivery of genome editing agents. Nature 553(7687):217-221

Jinek M, Chylinski K, Fonfara I, Hauer M, Doudna JA, Charpentier E (2012) A programmable dual-RNA-guided DNA endonuclease in adaptive bacterial immunity. Science 337(6096):816-21

Kim S, Kim D, Cho SW, Kim J, Kim JS (2014) Highly efficient RNA-guided genome editing in human cells via delivery of purified Cas9 ribonucleoproteins. Genome Res 24(6):1012-9

Ko TP, Liao CC, Ku WY, Chak KF, Yuan HS (1999) The crystal structure of the DNase domain of colicin E7 in complex with its inhibitor $\operatorname{Im} 7$ protein. Structure 7(1):91-102

Koonin EV, Makarova KS, Zhang F (2017) Diversity, classification and evolution of CRISPR-Cas systems. Curr Opin Microbiol 37:67-78

Lee K, Conboy M, Park HM, Jiang F, Kim HJ, Dewitt MA, Mackley VA, Chang K, Rao A, Skinner C, Shobha T, Mehdipour M, Liu H, Huang WC, Lan F, Bray NL, Li S, Corn JE, Kataoka K, Doudna JA, Conboy I, Murthy N (2017) Nanoparticle delivery of Cas9 ribonucleoprotein and donor DNA in vivo induces homology-directed DNA repair. Nat Biomed Eng 1:889-901

Qing G, Ma LC, Khorchid A, Swapna GV, Mal TK, Takayama MM, Xia B, Phadtare S, Ke H, Acton T, Montelione GT, Ikura M, Inouye M (2004) Cold-shock induced high-yield protein production in Escherichia coli. Nat Biotechnol 22(7):877-82

Schumann K, Lin S, Boyer E, Simeonov DR, Subramaniam M, Gate RE, Haliburton GE, Ye CJ, Bluestone JA, Doudna JA, Marson A (2015) Generation of knock-in primary human T cells using Cas9 ribonucleoproteins. Proc Natl Acad Sci U S A 112(33):10437-42

Scott DA, Zhang F (2017) Implications of human genetic variation in CRISPR-based therapeutic genome editing. Nat Med 23(9):1095-1101

Staahl BT, Benekareddy M, Coulon-Bainier C, Banfal AA, Floor SN, Sabo JK, Urnes C, Munares GA, Ghosh A, Doudna JA (2017) Efficient genome editing in the mouse brain by local delivery of engineered Cas9 ribonucleoprotein complexes. Nat Biotechnol 35(5):431-434

Vassylyeva MN, Klyuyev S, Vassylyev AD, Wesson H, Zhang Z, Renfrow MB, Wang H, Higgins NP, Chow LT, Vassylyev DG (2017) Efficient, ultra-high-affinity chromatography in a one-step purification of complex proteins. Proc Natl Acad Sci U S A 114(26):E5138-e5147

Li WQ, Qiao J, Liu Y and Ma LX (2018) Direct preparation of Cas9 ribonucleoprotein from E. coli for PCR-free seamless DNA assembly. Biorxiv doi:https://doi.org/10.1101/328468

Wu Q, Park A, Yang J, Suresh S, Bizhanova A, Gupta A, Bolukbasi MF, Walsh S, Bogorad RL, Gao G, Weng Z, Dong Y, Koteliansky V, Wolfe SA, Langer R, Xue W, Anderson DG (2016) Nat Biotechnol 3:328-333.

Yin H, Song CQ, Dorkin JR, Zhu LJ, Li Y (2016) Therapeutic genome editing by combined viral and non-viral delivery of CRISPR system components in vivo. Nat Biotechnol 34:328-333. 
Yin H, Xue W, Chen S, Bogorad RL, Benedetti E, Grompe M, Koteliansky V, Sharp PA, Jacks T, Anderson DG (2014) Genome editing with Cas9 in adult mice corrects a disease mutation and phenotype. Nat Biotechnol 32(6):551-3

Yu X, Liang X (2016) Improved delivery of Cas9 protein/gRNA complexes using lipofectamine CRISPRMAX. Biotechnol Lett 38:19-929.

Zetsche B, Gootenberg JS, Abudayyeh OO, Slaymaker IM, Makarova KS, Essletzbichler P, Volz SE, Joung J, van der Oost J, Regev A, Koonin EV, Zhang F (2015) Cpf1 is a single RNA-guided endonuclease of a class 2 CRISPR-Cas system. Cell 163(3):759-71

\section{Figure Legends}

Fig 1. An engineered cold-shock pCold I vector was harnessed to achieve co-expression of CL7-Cas9 and gRNA in E. coli. The CL7-Cas9 enzyme and gRNA were self-assembled in vivo to form CL7-Cas9 RNP. The stable Cas9 RNP can be easily obtained by one-step purification and in-column cleavage of the CL7 tag.

Fig 2. 12\% SDS-PAGE of purified Cas RNP by Ni-NTA or Im7 affinity columns.

Fig 3. Endonuclease activity assays of purified Cas RNP. (a) In vitro cleavage of target plasmid I (a single cutting site) by Cas9 RNP or CL7-Cas9 RNP, as well as target plasmid II (two cutting sites) by Cas12a RNP. (b) Delivery of Cas9 RNP and donor dsDNA in BFP-HEK293 cells can induce HDR to transfer them into GFP-HEK 293 Cells. (c) The bright-filed and fluorescent images of BFP-HEK293 cells after delivering Cas9 RNP (left panel), donor dsDNA (middle panel) and Cas9 RNP together with donor dsDNA (right panel) by lipofectamine CRISPRMAX in 48 hours later. (d) HDR efficiency was determined by GFP expression due to BFP editing. In total, $20 \%$ efficiency was observed when using Cas9 RNP/dsDNA comparing to $1 \%$ efficiency with addition of dsDNA alone. The previous values in refence 18 and 19 were also present here, indicating that the Cas9 RNP prepared by our method has comparable HDR editing efficiency. 
pCold CL7-Cas9 co-expression plasmid

$\underbrace{\operatorname{tac}}_{\text {CL7 tagged Cas9 protein expression element }}$\begin{tabular}{|c|c|c|c|c|}
$\operatorname{cspA} 5^{\prime}-U T R$ & CL7 & Cas9-His & cspA 3'-UTR \\
\hline
\end{tabular}

CL7 tagged Cas9 protein expression element guide RNA transcription element
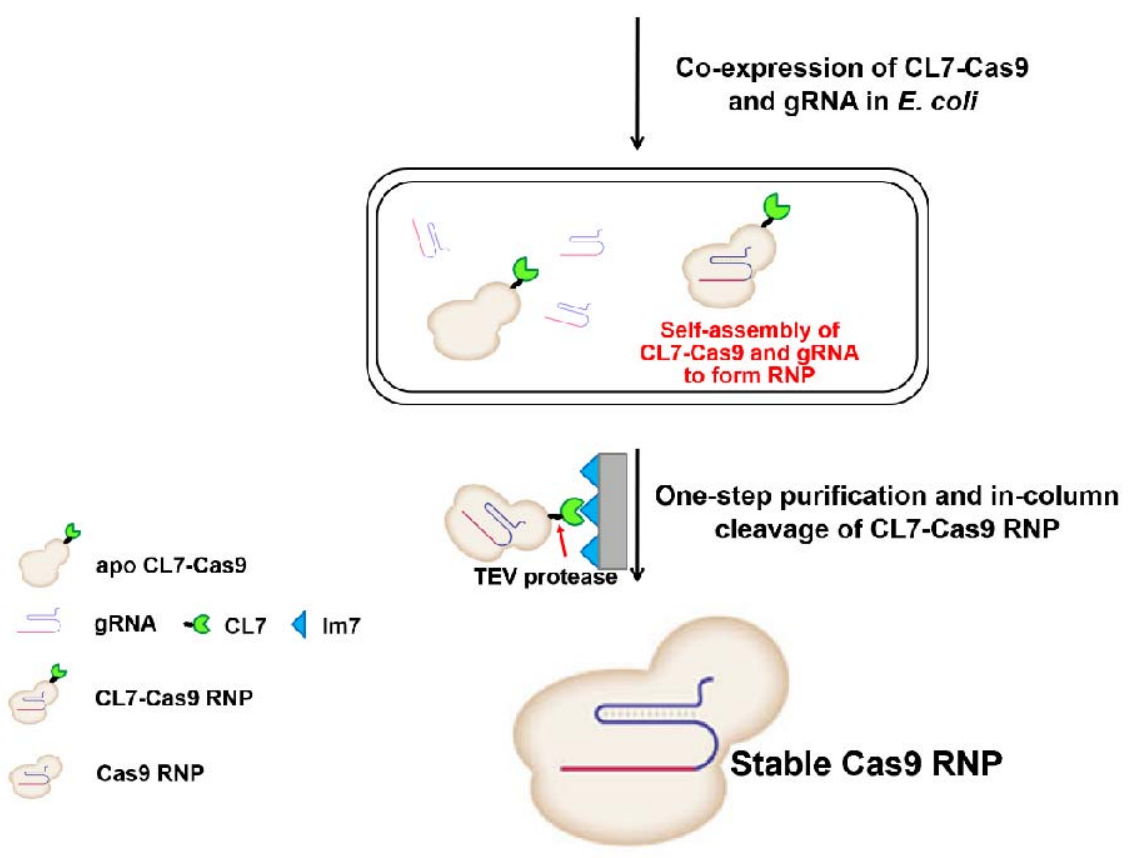

Fig. 1. 


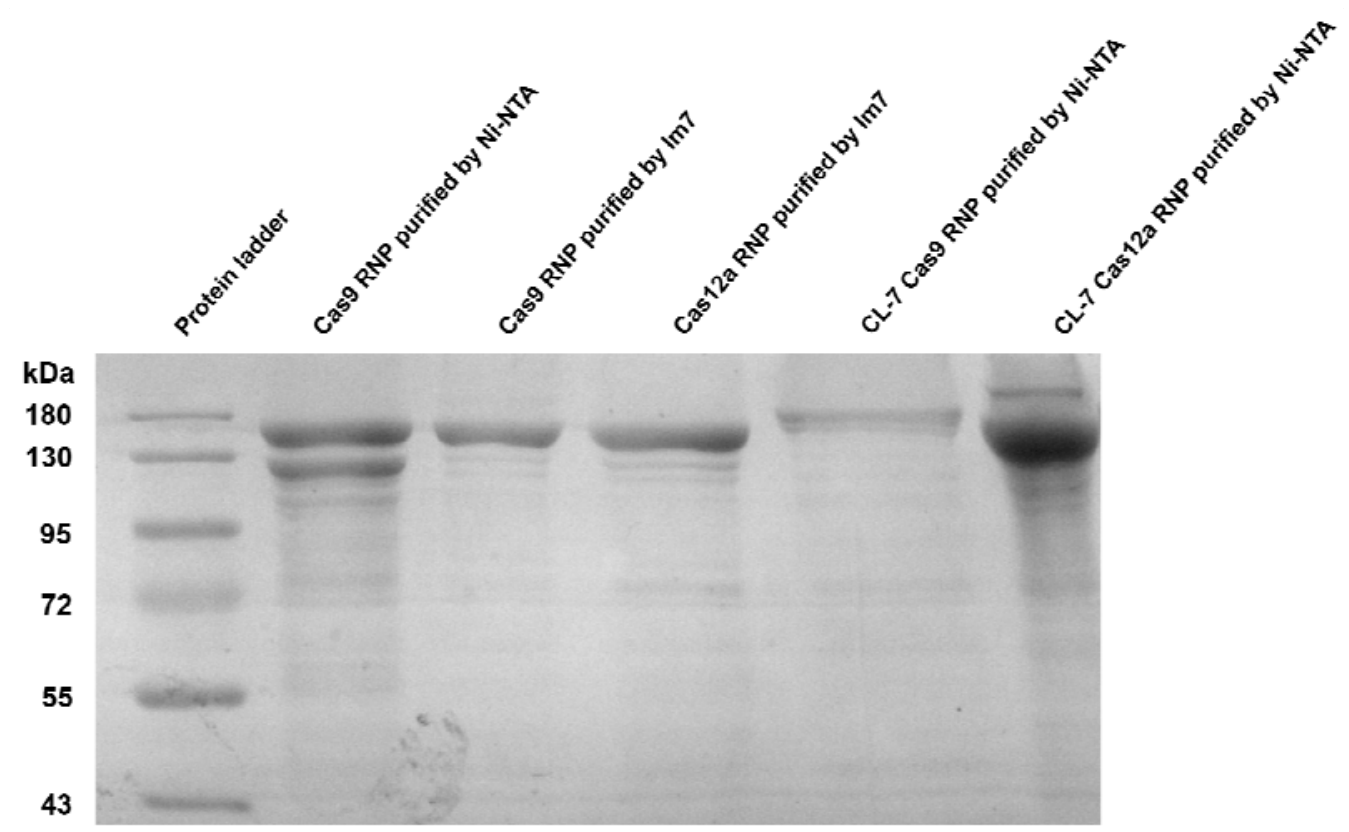

Fig. 2.

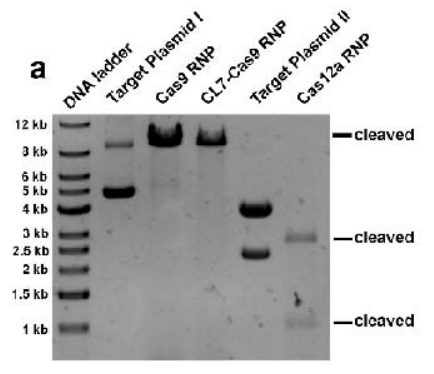

b

c
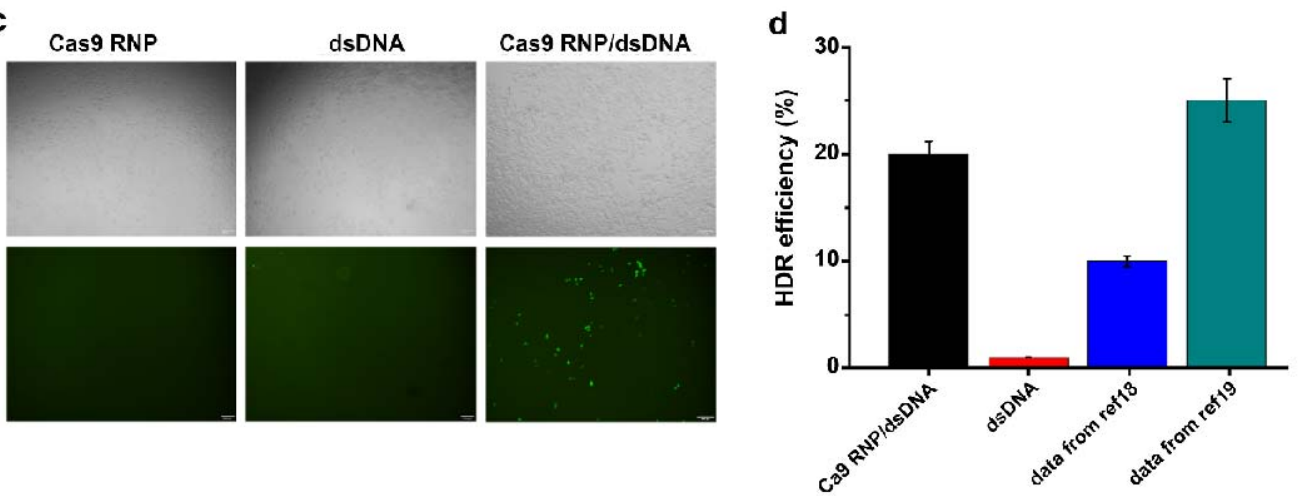

Fig. 3. 
bioRxiv preprint doi: https://doi.org/10.1101/377366; this version posted July 26, 2018. The copyright holder for this preprint (which was not certified by peer review) is the author/funder. All rights reserved. No reuse allowed without permission. 